\title{
Aeroacoustic instability analysis of a Helmholtz resonator using a High-Order DG Solver based on URANS equations
}

\author{
L.Criscuolo* \\ KU Leuven, Department of Mechanical Engineering, Celestijnenlaan 300, 3001 Leuven, Belgium \\ Flanders Make, DMMS Core Lab, Belgium \\ F. Renac ${ }^{\dagger}$, V. Couaillier ${ }^{\ddagger}$ \\ ONERA The French Aerospace Lab, 9320 Châtillon Cedex, France \\ H. Denayer ${ }^{\S}$, W. De Roeck ${ }^{\text {II }}$, W. Desmet " \\ KU Leuven, Department of Mechanical Engineering, Celestijnenlaan 300, 3001 Leuven, Belgium \\ Flanders Make, DMMS Core Lab, Belgium
}

\begin{abstract}
This paper presents an analysis of the aeroacoustic instabilities in a slit-type Helmholtz resonator subjected to a low Mach number grazing flow. This configuration can be susceptible to non-linear feedback phenomena due to the interaction between the hydrodynamic and acoustic field in the orifice proximity. The aeroacoustic flow field is simulated by solving the 2D unsteady Reynolds averaged Navier-Stokes (URANS) equations using a high-order discontinuous Galerkin (DG) solver. The response of the Helmholtz resonator to an incident acoustic field is analyzed using a two-port characterization technique. The onset of the aeroacoustic instabilities is investigated by applying an acoustic energy criterion based on the scattering matrix formulation. The numerical results are compared to experimental data obtained in a measurement campaign carried out at the KU Leuven test facility. The comparison between the numerical and experimental data demonstrates a very good agreement, highlighting the potential of the applied numerical approach for the aeroacoustic instabilities investigation.
\end{abstract}

\section{Introduction}

A EROACOUSTIC feedback phenomena can lead to high pressure fluctuations, self-sustained tonal acoustic oscillations, and structural vibrations in flow duct networks. These phenomena are typically triggered, under certain flow conditions, by the presence of duct singularities such as orifices, sudden area expansions or constrictions, valves, and cavities [1]. An understanding of the complex interactions involved in aeroacoustic instabilities is therefore of practical interest for many engineering applications in the automotive, aeronautical, and aerospace sectors. Previous studies have investigated flow-excited acoustic phenomena in resonant cavities [2-4], orifice plates [5- -9] or gas pipe networks [10-12]. In this work, the aeroacoustic instabilities in a Helmholtz resonator mounted in a duct system and subjected to a grazing flow at low Mach number are numerically and experimentally studied.

Helmholtz resonators are integrated into aircraft nacelle liners, automotive mufflers, ventilation and exhaust systems, in order control the noise propagation. In grazing flow conditions, the vortical flow disturbances, generated in the detached shear layer over the orifice, interact with the impinging acoustic waves. Depending on the flow conditions, the geometric parameters, and the frequency of the acoustic disturbances, the interaction between the hydrodynamic and the acoustic field can lead to an acoustic energy dissipation or amplification. In the case of a resonant cavity, acoustic resonances can influence the constructive reinforcement of the unstable vortical structures resulting in a risk of self-sustained acoustic instabilities. As discussed in [1, 10, 13] the aeroacoustic instabilities develop from a linear state to a non-linear regime as the damping mechanisms of vortex shedding become significant for the saturation of the shear layer response. Numerical methods based on linearized Navier-Stokes (LNS) equations can therefore be applied

\footnotetext{
*Ph.D. candidate, Mechanical Engineering Department, KU Leuven. DMMS lab, Flanders Make. luigi.criscuolo@kuleuven.be

$\dagger$ Senior Research Engineer, ONERA.

${ }^{\dagger}$ Head of the research unit Numerical Methods Group, ONERA.

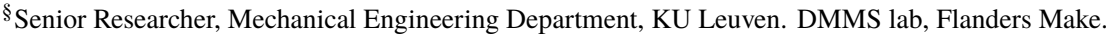

II Professor, Mechanical Engineering Department, KU Leuven.

" Full Professor, Mechanical Engineering Department, KU Leuven. DMMS lab, Flanders Make.
} 


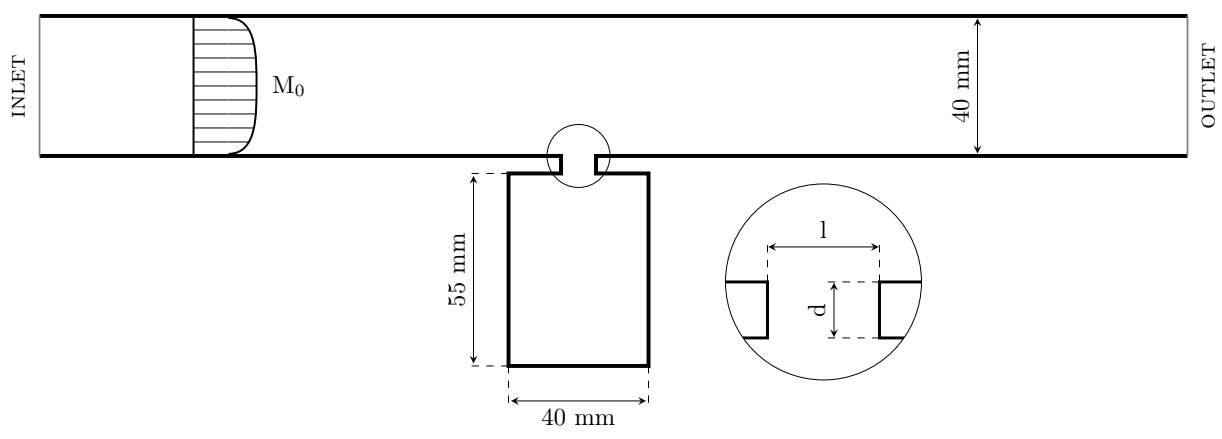

Fig. 1 Schematic description of the Helmholtz resonator.

to predict the onset of aeroacoustic instabilities in T-junctions [14], orifices [7] or Helmholtz resonators [15]. The non-linear aeroacoustic interactions can be modeled using scale-resolving methods such as direct numerical simulations (DNS) [16], and large-eddy simulations (LES) [9, 11]. However, these methods remain computationally demanding for wall-bounded flow applications.

In this paper, we obtained the aeroacoustic flow field by solving the unsteady Reynolds averaged Navier-Stokes equations coupled with turbulence transport equations using the high-order DG solver Aghora [17], developed by ONERA. This formulation proposes to capture the non-linear interaction between the vortical structures and the acoustic waves, providing an efficient alternative to more costly scale-resolving methods. The results are compared to measurement data, obtained from a dedicated experimental campaign on the open-circuit KU Leuven test facility. To facilitate this comparison, a two-port model is derived from both the numerical and the experimental results. Consequently, the acoustic energy criterion [8] is applied to this model to estimate the unstable frequency ranges of acoustic power amplification. The paper is outlined as follows. Section II provides the problem description, followed by a brief introduction to the acoustic two-port characterization framework in Section III. The results are shown and discussed in Section IV. Section V summarizes the main conclusions.

\section{Problem description}

The Helmholtz resonator analyzed in this work is schematically depicted in Fig 1 This two-dimensional geometry refers to a configuration previously investigated in Refs. [15, 18]. The resonator consists of a rectangular cavity with a length $1_{\mathrm{x}}=40 \mathrm{~mm}$, a depth $\mathrm{l}_{\mathrm{y}}=55 \mathrm{~mm}$ connected to a duct by an orifice. The orifice length is $1=10 \mathrm{~mm}$ and the neck depth is $\mathrm{d}=5 \mathrm{~mm}$. The main duct has an height of $\mathrm{H}=40 \mathrm{~mm}$, and a total length $\mathrm{L}=750 \mathrm{~mm}$. The computational domain considers this two-dimensional configuration and the experimental test rig design is obtained extruding the two-dimensional geometry over $\mathrm{W}=90 \mathrm{~mm}$ in the third dimension. The resonator is investigated considering flow conditions at Mach number $\mathrm{M}_{0}=0.074$ imposed at the main duct inlet, for which whistling phenomena have been experimentally observed. In the following sections, the numerical and the experimental setup are described in more detail.

\section{A. Numerical setup}

The high order DG software used in the simulations is Aghora [17], developed by ONERA. It is able to perform steady and unsteady flow simulations by solving compressible Navier-Stokes (N-S) equations with Reynolds averaged Navier-Stokes (RANS), large-eddy simulation (LES) or direct numerical simulation (DNS) modeling. The space discretization is based on a high-order discontinuous Galerkin method. High-order explicit and implicit Runge-Kutta methods are implemented for time integration.

In this work, the unsteady aeroacoustic flow field of the Helmholtz resonator is obtained by using RANS equations coupled with the one-equation turbulence model of Spalart-Allmaras [19]. Third-order accurate schemes $(\mathrm{p}=2)$ are used on the structured grid with 77014 quadrangles for the 2D simulations. Local mesh refinements are applied at the proximity of the walls and in the shear layer region. The numerical fluxes are evaluated with the Roe method and Harten entropy fix for the convective fluxes. We used the method of Bassi and coworkers [20] for the discretization of the diffusive terms. The time integration is performed with a strongly S-stable diagonally implicit Runge-Kutta (DIRK) scheme [21]. The non-linear algebraic system obtained at each stage is solved by using a fast non-linear LU-based 


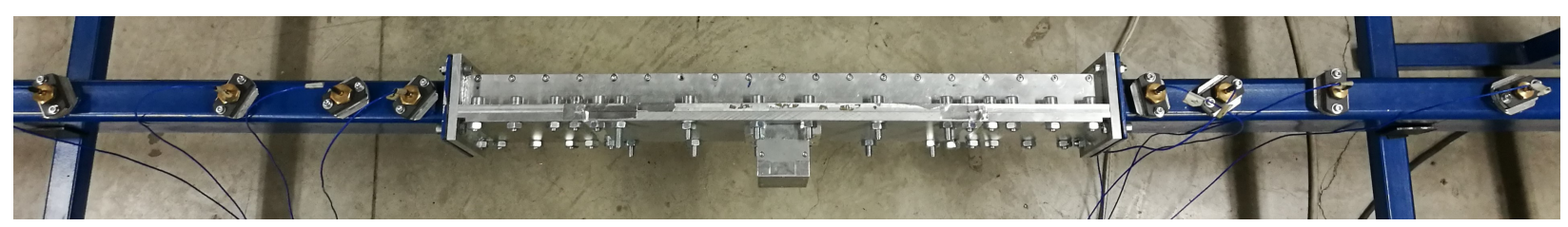

Fig. 2 Test object in the experimental setup.

solver [22], thus allowing the use of large time steps. We refer the readers to Ref. [17] for more details about the DG formulation and the Aghora code specifications.

No-slip and adiabatic conditions are applied at the walls. The inflow is subsonic $\left(\mathrm{M}_{0}=0.074\right)$ with uniform distribution of the total pressure $p_{i, t o t}$ and total temperature $T_{i, t o t}$. In order to match the experimental test conditions, the inflow turbulence intensity is set to $5 \%$ and the outlet static pressure to $p / p_{i, t o t}=0.994$. As a steady flow field solution is obtained, an acoustic pressure perturbation is injected from the inlet or the outlet boundary of the domain. The excitation signal has a Gaussian shape in space to avoid the Runge's phenomenon in the high-order polynomial description. The amplitude corresponds to a sound pressure level of $S P L=120 \mathrm{~dB}$. Two simulations have been carried out, exciting the system once from the inlet and once from the outlet, to enable the acoustic two-port characterization of the resonator using the two-source method, as described in Section III. Non-reflecting boundary conditions are imposed at the inlet and at the outlet of the duct to avoid end reflections [23, 24]. The reference states for the non-reflecting conditions are obtained from the previous RANS simulations. The unsteady simulations are run until all the energy has left the computational domain $(t=0.02 \mathrm{~s})$. The time signals of the flow parameters (density, velocity, and pressure) are stored in 30 monitoring points distributed in the upstream and downstream duct: 15 points per each duct, placed respectively along the longitudinal axis from $40 \mathrm{~mm}$ to $300 \mathrm{~mm}$ to the resonator orifice. The acoustic pressure perturbations are obtained subtracting the steady flow field from the perturbed field, and the frequency spectra are computed applying the FFT algorithm. The pressure spectra are then used to compute the transmission and reflection coefficients of the resonator by means of the two-port technique.

\section{B. Experimental setup}

The experimental campaign for the analysis of the aeroacoustic instabilities in the Helmholtz resonator has been carried out on the open circuit aeroacoustic test facility at KU Leuven [18]. Figure 2] shows the test object mounted between an inlet and an outlet duct with a rectangular cross-section of 90 by $40 \mathrm{~mm}$. Each of these ducts is equipped with 4 flush-mounted 1/4" microphones (type PCB 378C10), located respectively at $425 \mathrm{~mm}, 506 \mathrm{~mm}, 637 \mathrm{~mm}$ and 849 $\mathrm{mm}$ from the center of the orifice. An in-line loudspeaker is installed at the extremities of the measurement ducts to provide the acoustic excitation. At the exhaust of the downstream duct, various types of termination can be mounted to change the acoustic load. The upstream duct is connected to a flow generating unit, consisting of a frequency-regulated roots blower and an after-cooler. Measurements have been carried out imposing the inlet flow velocity at $\mathrm{M}_{0}=0.074$, continuously monitoring the temperature and the volume flow rate using a vortex-flow meter.

The data acquisition has been done using a LMS SCADAS III system, in combination with LMS Test.Lab rev.15. The two-port characterization has been carried out following a combined multiple source and multiple load approach, as explained in Section III, resulting in 4 independent datasets for each case. All the experiments have been performed with a stepped sine excitation in a frequency range from $100 \mathrm{~Hz}$ to $1880 \mathrm{~Hz}$. The frequency resolution is set to $10 \mathrm{~Hz}$ around the expected resonance frequency, gradually enlarging in the remainder of the frequency range. All autopower and crosspower spectra are computed using Welch's averaging technique with 60 averages and 25\% overlap [25].

\section{Acoustic characterization two-port method}

The aeroacoustic properties of the Helmholtz resonator are characterized using the N-port modeling framework [26] (Fig. 33. The resonator is considered as the acoustic element of a duct system, placed between inlet and outlet duct. It is treated as a two-port linear time-invariant system relating an input to an output. The methodology is independent of the reflection properties of the upstream and downstream duct terminations, allowing a direct comparison between numerical and experimental data. Even in presence of non-linear phenomena in the aeroacoustic field, the N-port methodology has been used to predict the whistling frequency of T-junctions [13, 14] and orifices [5, 27], applying the acoustic energy criterion based on the scattering matrix formulation [8]. 


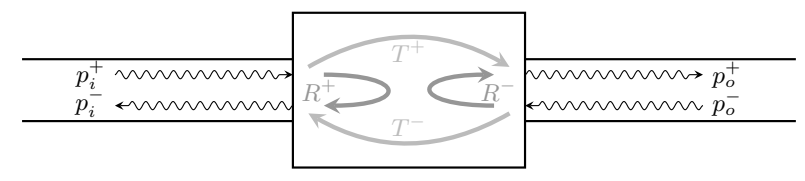

Fig. 3 Acoustic two-port model.

The acoustic two-port model makes abstraction of the physics inside the acoustic element describing the transmission and the reflection of the propagating sound using a linear matrix relation in frequency domain [28]. The model assumes that only plane waves propagate in the ducts and all high-order modes are evanescent. The acoustic field can, therefore, be described with only two independent variables, such as the acoustic pressure and velocity or the complex amplitudes of the upstream and downstream propagating wave. The scattering matrix formulation defines the linear relation between the complex amplitudes of the downstream $\left(p^{+}\right)$and upstream $\left(p^{-}\right)$propagating pressure wave at the inlet $\left(\bullet_{i}\right)$ and outlet $\left(\bullet_{o}\right)$ of the acoustic element, describing the acoustic behavior at an angular velocity $\omega$ as follows:

$$
\left\{\begin{array}{l}
p_{o}^{+}(\omega) \\
p_{i}^{-}(\omega)
\end{array}\right\}=\left[\begin{array}{ll}
T^{+}(\omega) & R^{-}(\omega) \\
R^{+}(\omega) & T^{-}(\omega)
\end{array}\right]\left\{\begin{array}{l}
p_{i}^{+}(\omega) \\
p_{o}^{-}(\omega)
\end{array}\right\}
$$

The scattering matrix $\underline{\underline{S}}$ contains the coefficients $T^{+}, T^{-}, R^{+}$and $R^{-}$, which represent, respectively, the transmission and reflection coefficients for the downstream $\left(\bullet^{+}\right)$and upstream $\left(\bullet^{-}\right)$propagating waves. Assuming a plane wave propagation, the validity of the model is restricted to frequencies below the cut-off frequency of the first transversal mode in the inlet and outlet duct. It corresponds to $f=1880 \mathrm{~Hz}$ for the geometry under consideration. The scattering matrix determination requires at least two independent states of the system. This can be achieved by varying the position of the excitation [29] (two-source method), by modifying the downstream impedance [30] (two-load method) or by a combination of both [31]. The scattering matrix is then computed as:

$$
\underline{\underline{S}}=\left[\begin{array}{ll}
T^{+}(\omega) & R^{-}(\omega) \\
R^{+}(\omega) & T^{-}(\omega)
\end{array}\right]=\left[\begin{array}{ll}
p_{o, \mathrm{I}}^{+}(\omega) & p_{o, \mathrm{II}}^{+}(\omega) \\
p_{i, \mathrm{I}}^{-}(\omega) & p_{i, \mathrm{II}}^{-}(\omega)
\end{array}\right]\left[\begin{array}{ll}
p_{i, \mathrm{I}}^{+}(\omega) & p_{i, \mathrm{II}}^{+}(\omega) \\
p_{o, \mathrm{I}}^{-}(\omega) & p_{o, \mathrm{II}}^{-}(\omega)
\end{array}\right]^{-1}
$$

where the Roman number refers to the two independent states. The use of more than two states, using a Moore-Penrose pseudoinverse for the matrix inversion, can improve the accuracy of the solution in an experimental setting by suppressing the influence of randomly distributed errors.

The complex amplitudes $p^{ \pm}$are computed by mapping the pressure spectra $p^{\prime}$ at $n \geq 2$ distinct positions in the duct on the analytical expression for the acoustic pressure field [32]:

$$
\left\{\begin{array}{l}
p^{+}(\omega) \\
p^{-}(\omega)
\end{array}\right\}=\left[\begin{array}{cc}
e^{-j k^{+} z_{1}} & e^{j k^{-} z_{1}} \\
\vdots & \vdots \\
e^{-j k^{+} z_{n}} & e^{j k^{-} z_{n}}
\end{array}\right]^{\otimes}\left\{\begin{array}{c}
p^{\prime}\left(\omega, z_{1}\right) \\
\vdots \\
p^{\prime}\left(\omega, z_{n}\right)
\end{array}\right\}
$$

where $z$ is the axial position of the measurement points and the symbol $\bullet{ }^{\otimes}$ denotes the Moore-Penrose pseudoinverse. The axial wavenumber $k^{ \pm}$is computed assuming an ideal uniform flow with Mach number $\mathrm{M}_{0}$ according to $k^{ \pm}=$ $k_{0} /\left(1 \pm \mathrm{M}_{0}\right)$ [29]. The term $k_{0}=\omega / c_{0}$ is the wavenumber in a quiescent medium, depending on angular velocity $\omega$ and the speed of sound $c_{0}$. In the experiments, Eqs. (2) and (3) are reformulated in terms of transfer function to suppress the influence of aerodynamic pressure fluctuations. Moreover, the axial wavenumber $k^{ \pm}$expression derived by Weng and Bake [33] is used, accounting for the visco-thermal attenuation at the walls:

$$
k^{ \pm}=\frac{k_{0}}{\left(1 \pm \mathrm{M}_{0}\right)}\left(1+\frac{1-j}{R} \sqrt{\frac{v}{2 \omega\left(1 \pm \mathrm{M}_{0}\right)}}\left(1+\frac{\gamma-1}{\sqrt{\mathrm{Pr}}}\right)\right)
$$

where Pr is the Prandtl number, $v$ the kinematic viscosity, $\gamma$ the ratio of specific heat constants and $R$ is the hydraulic radius of the test rig duct. An iterative optimization procedure [34] is also applied to reduce the uncertainties on the estimation of the flow parameters exploiting the over determination of Eq. (3). 

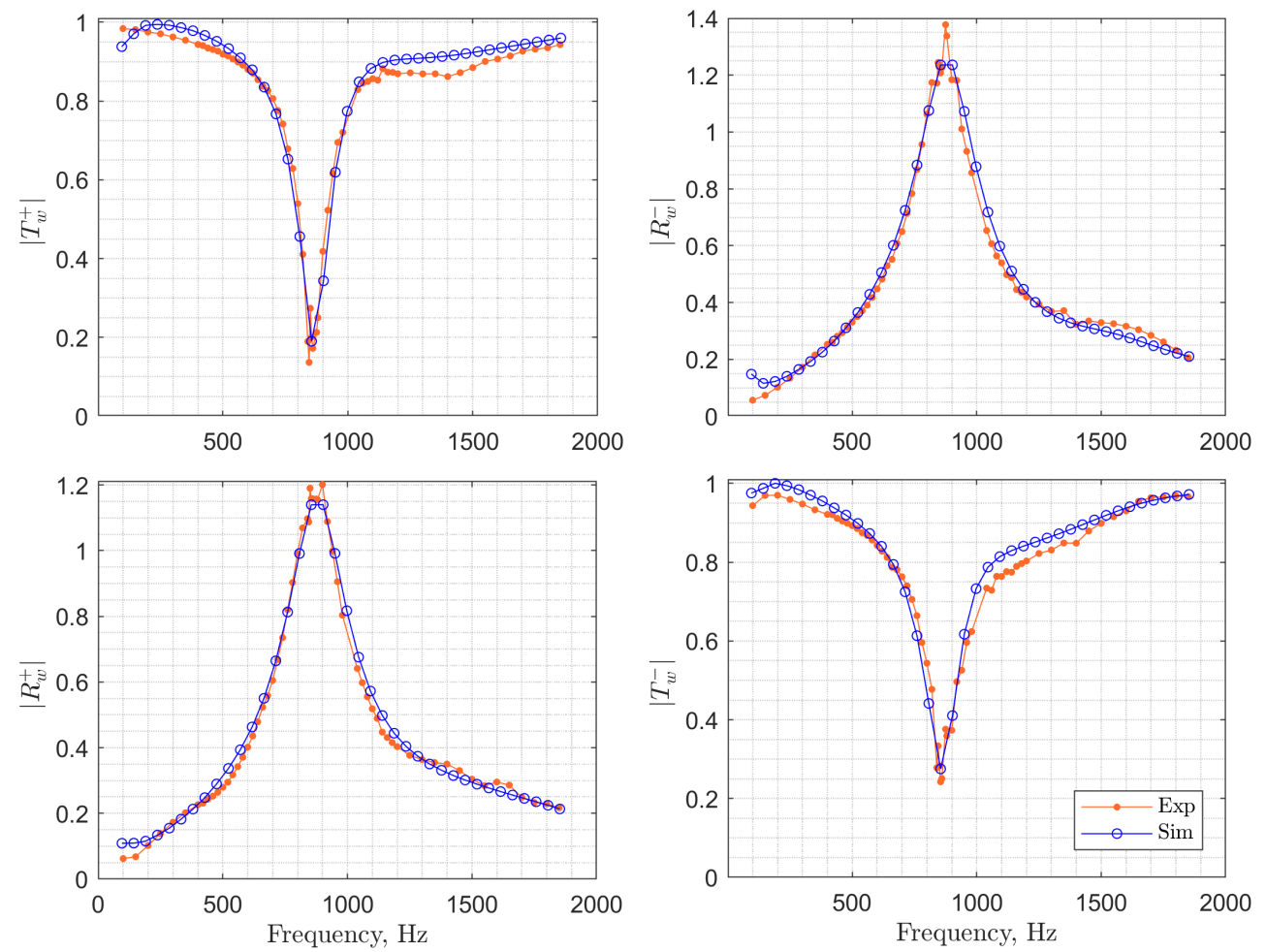

Fig. 4 Amplitude of the power scattering matrix coefficients. Orange filled circles: experimental results. Blue circles: numerical results.

The theoretical criterion for the prediction of the fluid-driven whistling potentiality of a duct component has been derived by Auregan and Starobinski in Ref. [8]. According to this formulation, the dissipated acoustic power $W_{\text {diss }}$ of a two-port element can be expressed as:

$$
W_{\text {diss }}=\sum_{i}\left(1-\lambda_{i}\right)\left|d_{i}\right|^{2}=\sum_{i} \xi_{i}\left|d_{i}\right|^{2}
$$

The terms $\lambda_{i}$ refer to the real positive eigenvalues of the matrix $\underline{\underline{S}}_{w}^{*} \underline{\underline{S}}_{w}$, where $\underline{\underline{S}}_{w}$ is the acoustic scattering matrix $\underline{\underline{S}}$ in energy form:

$$
\underline{\underline{S_{w}}}=\left[\begin{array}{cc}
T^{+}(\omega) & \frac{1+\mathrm{M}_{0}}{1-\mathrm{M}_{0}} R^{-}(\omega) \\
\frac{1-\mathrm{M}_{0}}{1+\mathrm{M}_{0}} R^{+}(\omega) & T^{-}(\omega)
\end{array}\right]
$$

The terms $\left|d_{i}\right|^{2}$ in Eq. (5) are derived from the eigenvectors of the matrix $\underline{\underline{S}}_{w}^{*} \underline{S}_{w}$ and are positive by definition. Hence, the so-called dissipation potentials $\xi_{i}=1-\lambda_{i}$ indicate the minimum and maximum sound power dissipation by the two-port element, considering all possible incident acoustic fields. The possible onset of whistling can therefore be analyzed considering the values of $\xi_{\min }=1-\lambda_{\max }$ : if the minimum dissipation potential $\xi_{\min }$ is negative, it indicates that the acoustic element can produce acoustic energy. On the other hand, a positive $\xi_{\min }$ guarantees an acoustic power dissipation.

\section{Results and Discussion}

In-house processing routines have been applied to the simulated and measured pressure spectra to carry out the plane wave decomposition and to compute the power scattering matrices and the dissipation potentials, as described in Section III. The amplitude of the simulated power scattering matrix coefficients is compared against experimental measurements in Fig 4 . The numerical predictions are in very good agreement with the experimental results. 


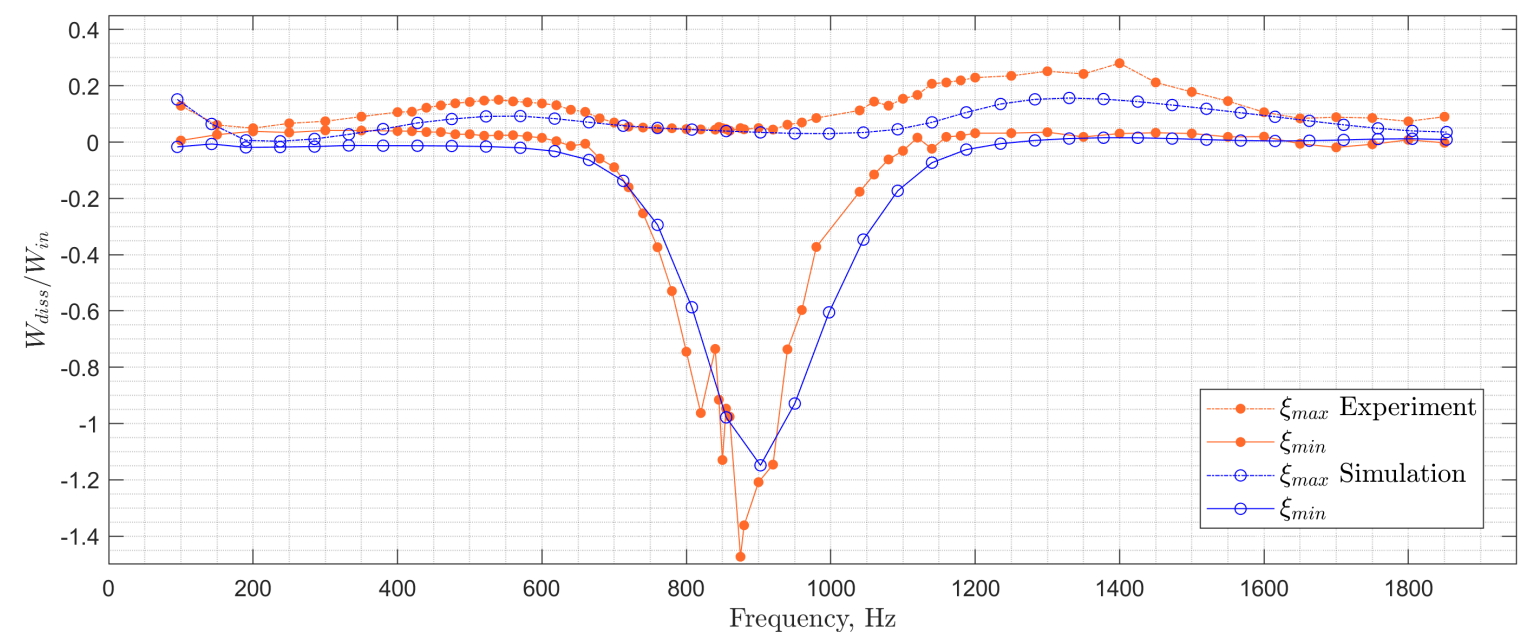

Fig. 5 Maximum and minimum of the normalized dissipated acoustic power. Orange filled circles: experimental results. Blue circles: numerical results.

As expected, the transmission coefficients show a minimum around the natural frequency of the resonator $f_{\text {res }}=855 \mathrm{~Hz}$, where the reflections have their maximum. In accordance with previous experimental work [18], the presence of a grazing flow over the resonator results in a small frequency shift of the resonance peak towards higher values compared to the no-flow case, $f_{\mathrm{M}=0}=845 \mathrm{~Hz}$. It is also worth noting that the reflection coefficients exceed the unit values around the resonance frequency, confirming the occurrence of flow-excited acoustic resonance phenomena. In particular, in this frequency range, the Helmholtz resonator is not behaving as a damping system but reflecting the incoming acoustic waves with amplified energy.

The results of the acoustic energy criterion for the high-order DG URANs simulations are reported and compared to the experiments in Fig 5. Both maximum and minimum dissipation potentials are in very good agreement with the experimental results. The minimum dissipation potentials $\xi_{\text {min }}$ predict a normalized acoustic power amplification between $f=700 \mathrm{~Hz}$ and $f=1150 \mathrm{~Hz}$, identifying the critical frequency range of potential onset of aeroacoustic instabilities. As explained in [1], an amplification of the acoustic wave potentially occurs when the convection velocity of the hydrodynamic disturbance across the orifice reasonably matches the oscillation period of the acoustic field. This condition corresponds to an integer multiple of the Strouhal number $S t=f l_{\text {ref }} / U_{c}$, where $l_{\text {ref }}$ is the orifice length and $U_{c}$ is the effective convection speed of the shear layer vorticity. Considering for the present case $U_{c} \simeq 9.58 \mathrm{~m} / \mathrm{s}$ based on the semi-empirical formula proposed in [4], it can be seen that the identified frequency interval of potential aeroacoustic instabilities satisfy the above condition around $S t \approx 1$, indicating the fluid-dynamic lock-in of the shear layer from $S t \simeq 0.73$ to $S t \simeq 1$. . In addition, the resonance acoustic mode of the cavity volume interact with this hydrodynamic shear layer instability modulating the frequency and the amplitude of the feedback, as discussed in [4, 35]. Specifically, the minimum of the dissipation potential $\xi_{\min }$ is observed at the resonance frequency, which suggests the important role of the resonator acoustic mode for the aeroacoustic instabilities. The onset of fluid-resonant phenomena in the analyzed Helmholtz resonator can, therefore, be accounted to the interaction between the first hydrodynamic instability mode of the shear layer and the acoustic resonance of the cavity.

The two-port model formulation is a valuable technique for the analysis of resonant flow-duct systems even when non-linear flow-acoustic phenomena occur. The adopted numerical approach demonstrates to correctly capture the aeroacoustic non-linear interaction in whistling conditions. Moreover, the two-dimensional assumption of the numerical model results accurate enough to predict the experimental observations.

\section{Conclusions}

The aeroacoustic instabilities in a Helmholtz resonator subjected to a low Mach number grazing flow are analyzed using a high-order DG solver based on URANS equations. The two-port characterization technique is employed to enable a direct comparison between the numerical results and experimental data obtained in a dedicated measurement campaign. The unstable frequency range of potential aeroacoustic instabilities onset is obtained applying an acoustic 
energy criterion. The results suggest the occurrence of a complex interaction between the fluid-dynamic instabilities of the shear layer and the acoustic mode of the resonator. The excellent agreement between the numerical results and the experimental data highlights the potential of the adopted approach for modeling the non-linear interaction between the hydrodynamic and the acoustic field, providing an efficient and accurate alternative to a more costly LES or DNS approach.

\section{Acknowledgments}

The research of L. Criscuolo is funded by an Early Stage Researcher grant within the European Project SSeMID Marie Curie Initial Training Network (GA 675008). The Research Fund KU Leuven is gratefully acknowledged for its support. L.Criscuolo also thanks Dr. Fabio Naddei for the fruitful discussions and his help with the ONERA solver Aghora.

\section{References}

[1] Ziada, S., and Lafon, P., "Flow-Excited Acoustic Resonance Excitation Mechanism, Design Guidelines, and Counter Measures," Applied Mechanics Reviews, Vol. 66, No. 1, 2013. https://doi.org/10.1115/1.4025788, 010802.

[2] Nelson, P. A., Halliwell, N., and Doak, P., "Fluid dynamics of a flow excited resonance, part I: Experiment," Journal of Sound and Vibration, Vol. 78, No. 1, 1981, pp. 15 - 38. https://doi.org/https://doi.org/10.1016/S0022-460X(81)80156-3

[3] Nelson, P. A., Halliwell, N. A., and Doak, P. E., "Fluid dynamics of a flow excited resonance, Part II: Flow acoustic interaction," Journal of Sound and Vibration, Vol. 91, 1983, pp. 375-402. https://doi.org/10.1016/0022-460X(83)90287-0

[4] Ma, R., Slaboch, P. E., and Morris, S. C., "Fluid mechanics of the flow-excited Helmholtz resonator," Journal of Fluid Mechanics, Vol. 623, 2009, pp. 1-26. https://doi.org/10.1017/S0022112008003911

[5] Kierkegaard, A., Boij, S., and Efraimsson, G., "A frequency domain linearized Navier-Stokes equations approach to acoustic propagation in flow ducts with sharp edges," The Journal of the Acoustical Society of America, Vol. 127, No. 2, 2010, pp. 710-719. https://doi.org/10.1121/1.3273899.

[6] Testud, P., Aurégan, Y., Moussou, P., and Hirschberg, A., "The whistling potentiality of an orifice in a confined flow using an energy criterion,” Journal of Sound and Vibration, Vol. 325, 2009, pp. 769-780. https://doi.org/10.1016/j.jsv.2009.03.046

[7] Kierkegaard, A., Allam, S., Efraimsson, G., and Åbom, M., "Simulations of whistling and the whistling potentiality of an in-duct orifice with linear aeroacoustics," Journal of Sound and Vibration, Vol. 331, No. 5, 2012, pp. 1084-1096. https://doi.org/10.1016/j.jsv.2011.10.028

[8] Aurégan, Y., and Starobinski, R., "Determination of Acoustical Energy Dissipation/Production Potentiality from the Acoustical Transfer Functions of a Multiport," Acta Acustica united with Acustica, Vol. 85, No. 6, 1999, pp. 788-792.

[9] Lacombe, R., Föller, S., Jasor, G., Polifke, W., Aurégan, Y., and Moussou, P., "Identification of aero-acoustic scattering matrices from large eddy simulation: Application to whistling orifices in duct," Journal of Sound and Vibration, Vol. 332, No. 20, 2013, pp. 5059-5067. https://doi.org/10.1016/j.jsv.2013.04.036

[10] Bruggeman, J. C., Hirschberg, A., van Dongen, M. E. H., Wijnands, A. P. J., and Gorter, J., "Self-sustained aero-acoustic pulsations in gas transport systems: Experimental study of the influence of closed side branches," Journal of Sound and Vibration, Vol. 150, No. 3, 1991, pp. 371-393. https://doi.org/10.1016/0022-460X(91)90893-O

[11] Föller, S., Polifke, W., and Tonon, D., "Aeroacoustic Characterization of T-Junctions Based on Large Eddy Simulation and System Identification," 16th AIAA/CEAS Aeroacoustics Conference, American Institute of Aeronautics and Astronautics, 2010. https://doi.org/10.2514/6.2010-3985

[12] Tonon, D., Moers, E., Golliard, J., and Hirschberg, A., "Aeroacoustics of a wall perforation in the pure grazing flow regime: effect of the perforation geometry," 18th AIAA/CEAS Aeroacoustics Conference (33rd AIAA Aeroacoustics Conference), American Institute of Aeronautics and Astronautics, 2012. https://doi.org/10.2514/6.2012-2054. AIAA 2012-2054.

[13] Karlsson, M., and Åbom, M., "On the Use of Linear Acoustic Multiports to Predict Whistling in Confined Flows," Acta Acustica united with Acustica, Vol. 97, 2011, pp. 24-33. https://doi.org/10.3813/AAA.918383

[14] Holmberg, A., Kierkegaard, A., and Weng, C., "A frequency domain linearized Navier-Stokes method including acoustic damping by eddy viscosity using RANS," Journal of Sound and Vibration, Vol. 346, 2015, pp. 229 - 247". https://doi.org/https: //doi.org/10.1016/j.jsv.2015.02.030 
[15] Criscuolo, L., Denayer, H., De Roeck, W., and Desmet, W., "Analysis of aeroacoustic instabilities in Helmholtz resonators using linear scattering-transfer matrices," 2018 AIAA/CEAS Aeroacoustics Conference, American Institute of Aeronautics and Astronautics, 2018. https://doi.org/10.2514/6.2018-3785, AIAA 2018-3785.

[16] Stein, L., Reiss, J., and Sesterhenn, J., "Numerical Simulation of a Resonant Cavity: Acoustical Response Under Grazing Turbulent Flow," New Results in Numerical and Experimental Fluid Mechanics XI, Springer International Publishing, Cham, 2018, pp. 671-681.

[17] Renac, F., de la Llave Plata, M., Martin, E., Chapelier, J. B., and Couaillier, V., Aghora: A High-Order DG Solver for Turbulent Flow Simulations, chapter and pages, pp. 315-335. https://doi.org/10.1007/978-3-319-12886-3_15.

[18] Criscuolo, L., Denayer, H., De Roeck, W., and Desmet, W., "Experimental analysis of aeroacoustic instabilities in Helmholtz resonators," Proceedings of the International Conference on Noise and Vibration Engineering ISMA 2018, KU Leuven, 2018, pp. 601-611.

[19] Spalart, P., and Allmaras, S., A one-equation turbulence model for aerodynamic flows, chapter and pages. https://doi.org/10. 2514/6.1992-439. URL https://arc.aiaa.org/doi/abs/10.2514/6.1992-439

[20] Bassi, F., Rebay, S., Mariotti, G., Pedinotti, S., and Savini, M., "A High-order accurate discontinuous finite element method for inviscid and viscous turbomachinery flows," Proceedings of the 2nd European Conference on Turbomachinery Fluid Dynamics and Thermodynamics, Decuypere, R. and Dibelius, G., 1997, pp. 99-108.

[21] Alexander, R., "Diagonally Implicit Runge-Kutta Methods for Stiff O.D.E.'s," SIAM Journal on Numerical Analysis, Vol. 14, No. 6, 1977, pp. 1006-1021. https://doi.org/10.1137/0714068 URL https://doi.org/10.1137/0714068

[22] Parsani, M., Ghorbaniasl, G., Lacor, C., and Turkel, E., "An implicit high-order spectral difference approach for large eddy simulation," J. Comput. Physics, Vol. 229, 2010, pp. 5373-5393.

[23] V.Couaillier, "Effective Multdimensional Non-Reflective Boundary Condition for CFD Calculations Applied to Turboengine Aeroacoustics Prediction," ISABE 2005-1185, 2005.

[24] Viviand, H., and Veuillot, J., "Methods pseudo-instationnnaires pour le calcul d'ecoulements transsoniques," ONERA publication 1980-5, 1978.

[25] Welch, P., "The use of fast Fourier transform for the estimation of power spectra: A method based on time averaging over short, modified periodograms," IEEE Transactions on Audio and Electroacoustics, Vol. 15, No. 2, 1967, pp. 70-73. https://doi.org/10.1109/tau.1967.1161901

[26] Åbom, M., "Measurement of the scattering-matrix of acoustical two-ports," Mechanical Systems and Signal Processing, Vol. 5, No. 2, 1991, pp. 89-104. https://doi.org/10.1016/0888-3270(91)90017-Y

[27] Sovardi, C., Jaensch, S., and Polifke, W., "Concurrent identification of aero-acoustic scattering and noise sources at a flow duct singularity in low Mach number flow," Journal of Sound and Vibration, Vol. 377, 2016, pp. 90-105. https://doi.org/10.1016/j.jsv.2016.05.025

[28] Munjal, M., Acoustics of Ducts and Mufflers with Application to Exhaust and Ventilation System Design, J.Wiley \& Sons, 1987.

[29] Munjal, M., and Doige, A., "Theory of a two source-location method for direct experimental evaluation of the fourpole parameters of an aeroacoustic element," Journal of Sound and Vibration, Vol. 141, No. 2, 1990, pp. 323-333. https://doi.org/10.1016/0022-460x(90)90843-o

[30] Lung, T. Y., and Doige, A., "A time-averaging transient testing method for acoustic properties of piping systems and mufflers with flow," The Journal of the Acoustical Society of America, Vol. 73, No. 3, 1983, pp. 867-876. https://doi.org/10.1121/1.389056

[31] Åbom, M., "A note on the experimental determination of acoustical two-port matrices," Journal of Sound and Vibration, Vol. 155, No. 1, 1992, pp. 185-188. https://doi.org/10.1016/0022-460x(92)90655-h.

[32] Fujimori, T., Sato, S., and Miura, H., "An automated measurement system of complex sound pressure reflection coefficients," Proceedings of Inter Noise, Vol. 84, 1984, pp. 1009-1014.

[33] Weng, C., and Bake, F., "An Analytical Model for Boundary Layer Attenuation of Acoustic Modes in Rigid Circular Ducts With Uniform Flow," Acta Acustica united with Acustica, Vol. 102, No. 6, 2016, pp. 1138-1141. https://doi.org/10.3813/aaa.919025.

[34] Denayer, H., "Flow-acoustic characterization of duct components using multi-port techniques," Ph.D. thesis, KU Leuven, 2017.

[35] Criscuolo, L., Denayer, H., De Roeck, W., and Desmet, W., "Experimental analysis of whistling in flow-excited Helmholtz resonators," ISMA 2020, KU Leuven, 2020. 